\title{
The Leser-Trelat Syndrome: About A Case
}

\author{
Assenhaji I*, Douhi Z, Dassouli R, Elloudi S, Baybay H, Mernissi FZ
}

Department of Dermatology at chu hassan II fes. Morocco

DOI: $10.36347 /$ sjmcr.2020.v08i05.020

| Received: 17.02.2020 | Accepted: 24.02.2020 | Published: 27.05.2020

*Corresponding author: Assenhaji louizi ibtissam

\section{Abstract}

HE LESER-TRELAT SYNDROME (LTS) is a paraneoplastic syndrome characterised by the sudden eruption and rapid increase in size and number of seborrhoeic keratoses as we are reporting in our case [1]. The exact underlying pathogenesis is unknown. Primarily affect the thorax and dorsum, followed by the extremities, face, abdomen, neck and axillaire. It is usually caused by malignancies such as gastrointestinal adenocarcinoma, but also lung, kidney, liver, or pancreatic cancer, mycosis fungoides, Sézary syndrome, and plasmacytoma, have been described in association with this paraneoplastic disorder [3, 4, 5]. After the removal of the internal neoplasm there is a regression of the seborrheic keratoses [2]. LTS should be careful investigated for the existence of an underlying malignancy as illustrated in our case which objectified a tumor of sigmoid.

Keywords: LESER-TRELAT SYNDROME (LTS), axillaire, seborrhoeic keratoses.

Copyright @ 2020: This is an open-access article distributed under the terms of the Creative Commons Attribution license which permits unrestricted use, distribution, and reproduction in any medium for non-commercial use (NonCommercial, or CC-BY-NC) provided the original author and source are credited.

\section{INTRODUCTION}

Seborrheic keratosis (SK) is a common benign epidermal tumor with predominance in adult patients. The Leser-Trelat syndrome (LTS) can evolve an association with an internal malignancy, as we are reporting in our case.

\section{Case Report}

An 83-year-old man, with no notable pathological history, hospitalized in the visceral surgery department. The patient presented for a year and a half lesions in the trunk, developed rapidly, over a period of 6 months. He also noticed for three months melenas with transit disorders, justifying his consultation or the diagnosis of sigmoid tumor was confirmed histologically.

Clinical examination revealed sessile exophytic tumors with a round or oval appearance, 1 to $3 \mathrm{~cm}$ in diameter, welldefined. The surface of the lesion was warty, brown-black in color with numerous black keratotic plugs [Figure 1, 2]. The dermoscopic examination showed a cerebral aspect, with pseudo cysts and pseudocomedons [Figure 3, 4], for which the diagnosis of seborrheic keratosis was made.

The extension balance was normal. The patient is a candidate for surgery
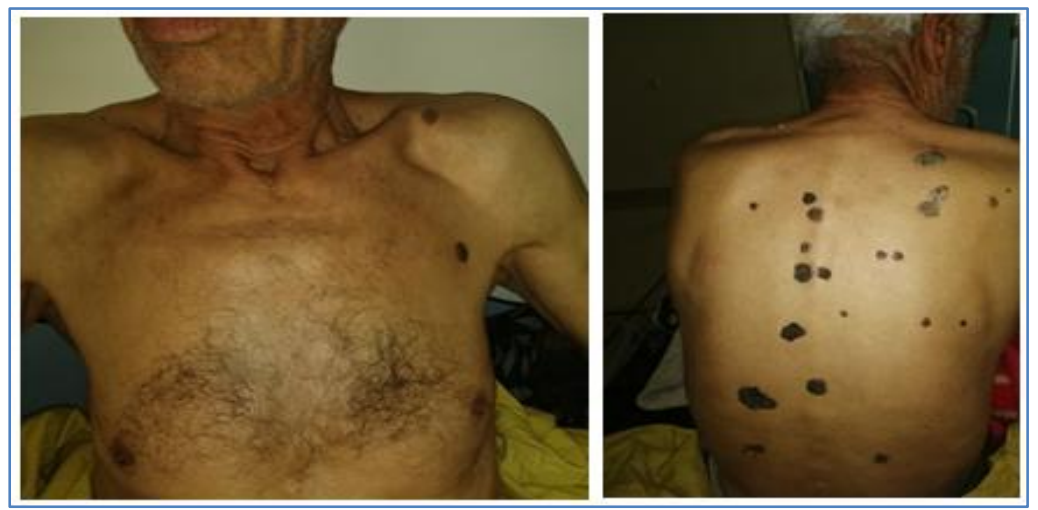

Fig-1, 2: Sessile exophytic tumors with a round or oval appearance, 1 to $3 \mathrm{~cm}$ in diameter, welldefined. The surface of the lesion was warty, brown-black in color 


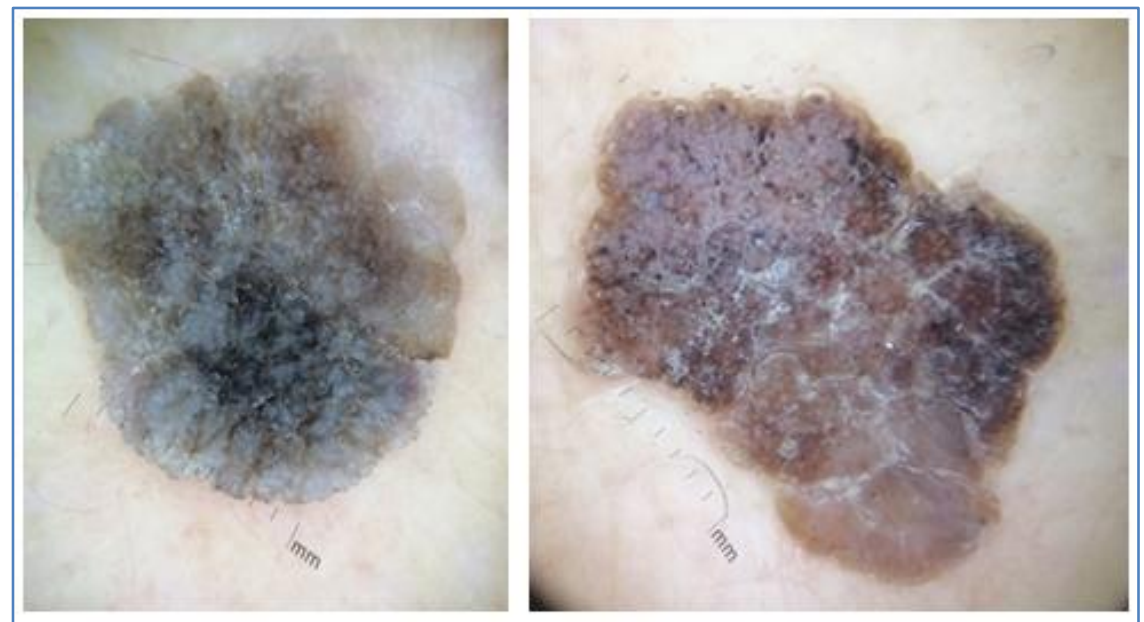

Fig-3, 4: The dermoscopic examination showed a cerebral aspect, with pseudo cysts and pseudocomedons

\section{DISCUSSION}

LTS is a paraneoplastic syndrome characterised by the sudden eruption and rapid increase in size and number of seborrhoeic keratoses [1]. Primarily affect the thorax and dorsum, followed by the extremities, face, abdomen, neck and axillaire. Pruritus and inflammation are frequent findings.

The exact underlying pathogenesis is unknown.The existence of Leser-Trelat syndrome is under debate and it is due to the high frequency of seborrheic keratosis in elderly people. More recent observations suggested that TGF-a produced by the internal malignancy could be responsible for the acute eruption of seborrheic keratosis [2].

It is usually caused by malignancies such as gastrointestinal adenocarcinoma, but also lung, kidney, liver, or pancreatic cancer, mycosis fungoides, Sézary syndrome, and plasmacytoma, have been described in association with this paraneoplastic disorder [3-5].

Physical examination with complete blood count, serum biochemistry, chest radiography, mammography, Pap smear, PSA screening, upper digestive endoscopy and colonoscopy are required during this investigation [6].

Curettage is the treatment of first choice. Other alternatives are laser therapy, cryosurgery, electrocautery or excision. After the removal of the internal neoplasm there is a regression of the seborrheic keratoses [2].

\section{Conclusion}

Leser Trelat syndrome is the development of large numbers of SKs is entirely dependent on the extremely rapid onset of these extensive lesions. It should be careful investigated for the existence of an underlying malignancy.

\section{REFERENCES}

1. Flugman SL, McClain SA, Clark RAF. Transient eruptive seborrheic keratoses associated with erythrodermic psoriasis and erythrodermic drug eruption: Report of two cases, J Am Acad Dermatol. 2001; 45: S212-4.

2. Heaphy Jr. MR, Millns JL, Schroeter AL. The sign of Leser-Trelat in a case of adenocarcinoma of the lung. J Am Acad Dermatol. 2000; 43: 386-90

3. Schwartz RA. Sign of Leser-Trelat, J Am Acad Dermatol. 1996; 35: 88-95.

4. Asri H, Soualhi M. The sign of leser-trélat: think in the adenocarcinoma of the lung. Pan Afr Med J. 2018;30:270.

5. Schneider R, Wollina U: Das Leser-TrelatSyndrom bei einem Plasmozytom. $Z$ Dermatol. 1998;186:106-8.

6. Pipkin CA, Lio PA. Cutaneous manifestations of internal malignancies: an overview. Dermatol Clin. 2008;26:1-15. 